\title{
VIII. A new specifying method for stress and strain in an elastic solid
}

\section{Lord Kelvin}

To cite this article: Lord Kelvin (1902) VIII. A new specifying method for stress and strain in an elastic solid, Philosophical Magazine Series 6, 3:13, 95-97, DOI: 10.1080/14786440209462741

To link to this article: http://dx.doi.org/10.1080/14786440209462741

Published online: 09 Jun 2010.

Submit your article to this journal $\longleftarrow$

Џll Article views: 3

Q View related articles $\square$ 
Case 2.-In passing from a narrow to a wide ruling in going from left to right.
Focus in.
Focus out.
Dark band.
Light band.

\section{Orders on the left of the Central Image.}

The results just given must be reversed.

The theoretical results so obtained were confirmed by observations on gratings with prominent errors. It had previously been noticed that when the telescope was focussed exactly on the surface of the grating the bands disappeared, by putting the telescope considerably out of focus the bands were clearly seen. Following the indications of the theory, and focussing first in and then out, it was seen that the dark bands changed to light and the light to dark. On comparing the images on the right and left of the central one, the bands were seen to be exactly reversed. The same effect was produced by the practically equivalent process of turning the $p$ ate upside down $*$.

In all the gratings that I have examined the principal error has been perjodic in character, so that the bands are distributed over the image with a certain amount of regularity.

In conclusion I beg to express my thanks to Lord Blythswood, the resources of whose laboratory have been at my disposal in examining the gratings.

Blythowood Laboratory, Renfrew, N.B.

VIII. A New Specifying Method for Stress and Strain in an Elastic Solid. By Lord Kelvin †.

THE method for specifying stress and strain hitherto followed by all writers on elasticity has the great disadvantage that it essentially reguires the strain to be infinitely

If we treat $F_{1}$ and $F_{2}$ as two sources of light as in Fresnel's interference experiment, the breadth of an interference-band at $B^{\prime \prime}$ would be $b \lambda / f \delta \theta$.

A simple calculation shows that in general this quantity is iarge compared with the distance within which there is overlapping, so that the inter/erence effect is comparatively insigniticant.

* I have devised an arrangement in connexion with I ord Blythswood's dividing-engine which makes it possible to cut any number of lines to the inch. Since the paper was written I have veified the results by using a plate containing two distinct rulings.

$t$ Communicated by the Author. 
small. As a notational method it has the inconvenience that the specifying elements are of two essentially different kinds (in the notation of Thomson and Tait $e, f, g$, simple elongations; $a, b, c$, shearings). Both these faults are avoided if we take the six lengths of the six edges of a tetrahedron of the solid, or what amounts to the same, though less simple, the three pairs of face-diagonals of a hexahedron*, as the. specifying elements. This I have thought of for the last thirty years, but not till to-day (Dec. 16) have I seen how to make it conveniently practicable, especially for application to the generalized dynamics of a erystal.

We shall suppose the solid to be a homogeneous crystal of any possible character. Cut from it a tetrahedron ABCD of any shape and orientation. Let the three non-intersecting pairs $(A B, C D),(B C, A D),(C A, B D)$ of its six edges be denoted by

$$
\left(f, f^{\prime \prime}\right), \quad\left(g, g^{\prime}\right), \quad\left(h, h^{\prime}\right) \ldots \ldots
$$

Parallel to the edge $A B$ apply to the finces $A D C, B D C$ equal and opposite pulls, $P$, equally distributed over them. These two balancing pulls we shall call a stress or a stress-component. Similarly, parallel to each of the five other edges apply balancing pulls on the pair of faces cutting it. Thus we have in all six stress-components parallel to the six edges of the tetrahedron, denoted as follows :-

$$
\left(\mathrm{P}, \mathrm{P}^{\prime}\right), \quad\left(\mathrm{Q}, \mathrm{Q}^{\prime}\right), \quad\left(\mathbf{R}, \mathbf{R}^{\prime}\right) \ldots
$$

and we suppose that these forces, applied as they are to the surface of the solid, are balanced in virtue of the mutual forces between its particles, when its edges are of the lengths specified as in (1). Let $f_{0}, f_{0}^{\prime}, g_{0}, g_{0}{ }^{\prime}, h_{0}, h_{0}{ }^{\prime}$, be the values of the specifying elements in (1) when no forces are applied to the faces. Thus the differences from these values, of the six lengths shown in formula (1), represent the strain of the substance when under the stress represented by (2).

Let $w$ be the work done when pulls upon the faces, each commencing at zero, are gradually increased to the values

* 'This name, signifying a figure bounded by three pairs of parallel planes, is admitted in crystallography; but the longer and less expressive "parallelepiped" is too frequently nsed instead of it by mathematical writers and teachers. A hexahedron with its angles acute and obtuse is what is commonly called, both in pure mathematics and crystallography, a rhombohedron. A right-angled hexahedron is a brick, for which no Greel or other learned name is hitherto to the front in usage. A rectangular equilateral hexahedron is a cube. 
shown in (2). In the course of this process we have

$$
d w=\mathrm{P} d f+\mathrm{P}^{\prime} d f^{\prime}+\mathrm{Q} d g+\mathrm{Q}^{\prime} d g^{\prime}+\mathrm{R} d h+\mathrm{R}^{\prime} d h^{\prime} \ldots
$$

Hence if we suppose $w$ expressed as a function of $f, f^{\prime}$, $g, g^{\prime}, h, h^{\prime}$, we have

$\frac{d w}{d f}=\mathrm{P}, \frac{d w}{d f^{\prime}}=\mathrm{P}^{\prime}, \frac{d w}{d g}=\mathrm{Q}, \frac{d w}{d g^{\prime}}=\mathrm{Q}^{\prime}, \frac{d w}{d h}=\mathrm{R}, \frac{d w}{d h^{\prime}}=\mathrm{R}^{\prime} \ldots$

This completes the foundation of the molar dynamics of an elastic solid of the most general possible kind according to Green's theory, expressed in terms of the new mode of specifying stresses and strains.

In a communication to the Royal Society of Edinburgh promised for Jan. 20, 1902, I hope to use with advantage this mode of specification in working out some details of the molecular dynamics of a crystal.

IX. Some General Theorems concerning Forced Vibrations and Resonance. By Lord RA YLEIGH, F.R.S.*

TTHE general equation for the small vibrations of a system whose configuration is defined by the generalized coordinates $\psi_{1}, \psi_{2}, \ldots$ may be written $\dagger$

$$
\frac{d d \mathrm{~T}}{d t} d \dot{\psi}+\frac{d \mathrm{~F}}{d \dot{\psi}}+\frac{d \mathrm{~V}}{d \dot{\psi}}=\Psi, \quad . \quad .
$$

where T, F, V, denoting respectively the kinetic energy, the dissipation function, and the potential energy, have the forms

$$
\left.\begin{array}{l}
\mathrm{T}=\frac{1}{2} a_{11} \dot{\psi}_{1}^{2}+\frac{1}{2} a_{22} \dot{\psi}_{2}^{2}+\ldots+a_{12} \dot{\psi}_{1} \dot{\psi}_{2}+\ldots \\
\mathrm{F}=\frac{1}{2} b_{11} \dot{\psi}_{1}^{2}+\frac{1}{2} b_{22} \dot{\psi}_{2}^{2}+\ldots+b_{12} \dot{\psi}_{1} \dot{\psi}_{2}+\ldots \\
\mathrm{V}=\frac{1}{2} c_{11} \boldsymbol{\psi}_{1}^{2}+\frac{1}{2} c_{22} \dot{\psi}_{2}{ }^{2}+\ldots+c_{12} \psi_{1} \psi_{2}+\ldots
\end{array}\right\},
$$

in which the coefficients $a, b, c$ are constants.

If we substitute in (1) the values of $T, F$, and $V$, and write $\mathrm{D}$ for $d / d t$, we obtain a system of equations which may be put into the form

$$
\left.\begin{array}{c}
e_{11} \psi_{1}+e_{12} \psi_{2}+e_{13} \psi_{3}+\ldots=\Psi_{1} \\
e_{21} \psi_{1}+e_{22} \psi_{2}+e_{23} \psi_{3}+\ldots=\Psi_{2} \\
e_{31} \psi_{1}+e_{32} \psi_{2}+e_{33} \psi_{3}+\ldots=\Psi_{3} \\
\cdot \cdot \cdot \cdot \cdot \cdot \cdot \cdot \cdot \cdot \cdot
\end{array}\right\},
$$

Phil. Mog. S. 6. Vol. 3. No. 13. Jan. 1902. 